\title{
CRYPTOCURRENCY FORECASTING USING OPTIMIZED SUPPORT VECTOR MACHINE WITH SINE COSINE METAHEURISTICS ALGORITHM
}

\author{
Mohamed Salb* \\ Ali Elsadai, \\ Miodrag Živković, \\ Nebojša Bačanin
}

Singidunum University,

Belgrade, Serbia

\begin{abstract}
:
Predicting the market behaviour is a crucial task for cryptocurrency investors. Based on the prediction, they make decisions that will either bring profit or loss. The prediction is typically involves the historical data that is used to forecast the future behaviour of the prices on the market.

Prediction is based on the machine learning approach. The nature-inspired algorithms have been successfully applied in optimization of numerous machine learning models in the recent years. Swarm intelligence metaheuristics, a family of nature-inspired algorithms, have proven to be excellent optimizers not only in the machine learning domain, but in various other practical domains as well. This paper proposes one such approach, more precisely the enhanced version of the sine cosine algorithm to optimize the support vector machine, and use it to predict the cryptocurrency prices. The basic SCA was improved with a simple exploration mechanism, and then compared to other approaches executed on the same datasets.

The results obtained from the performed experimental simulations indicate that the proposed method achieved better performances than other approaches included in the research.
\end{abstract}

Keywords:

Cryptocurrency, Blockchain, Machine Learning, Sine and Cosine Algorithm, Support Vector Machines.

\section{INTRODUCTION}

The biggest challenge that cryptocurrency investors face is predicting and forecasting the market behaviour and making the right decisions that bring high profit. Cryptocurrencies differ from other currencies by being independent and wholly based on peer-to-peer transactions. The possibility that cryptocurrencies can replace today's online payment methods encourages people to invest and support cryptocurrencies which provides more secure online transactions.

Financially, the market of cryptocurrency is called the stock market. Market prediction plays the primary role to maximize profit in the field of cryptocurrency. Therefore, the market prediction has grown and advanced significantly, including machine learning technologies to predict DIPs and HODLs [1]. 
Cryptocurrency is entirely on blockchain technology that makes it more secure and faster than any other system. The main advantages of blockchain, transactions are processed instantly, and people cannot trace them.

Bitcoin (BTC) is considered to be the leading cryptocurrency by the community. It requires a digital signature from all participated parties (sender and receiver) [2]. It uses cryptography techniques performed by networks to verify transactions and record them in a public distributed ledger known as a blockchain.

In 2011 Litecoin (LTC) was launched with a faster transaction rate as fast as less than 1 minute and enough intelligence to deal with more significant volumes than Bitcoin [3]. Litecoin creators developed network technology to ensure repeated block creation, and the block creation time was reduced. Before building a block, it takes 10 minutes to create a block, with Litecoin decreased to 2.5 minutes each block with a new hashing algorithm.

Ethereum is a decentralized, Turing-complete blockchain framework for building and executing intelligent contracts and circulating systems [4]. Ethereum has no limits on circulation, and it may be sold on cryptocurrency exchanges, not as one of the methods of payment, but only as part of the network of Ethereum [5].

NEM is also known as the 'peers' or P2P blockchain notarization, a network of computers joined and used to pay for online transactions and perform the online message function. NEM has a registered NEM version called Mijin and then makes NEM the first blockchain public or private combination [3].

Ripple is an open-source digital technology and a peer-to-peer (P2P) system created by Jed McCaleb and his colleague, Chris Larsen. The Ripple is governed by one institution. In its transactions, Ripple provides another safety mechanism. The Ripple development is based on the Byzantine Consensus Protocol, which now has a maximum of 100 million. One of the most remarkable things about the Ripple payment transaction is that it only takes 4 seconds [3] [6].

\subsection{OBJECTIVE}

This paper aims to use an enhanced since cosine algorithm (eSCA) to improve support vector machine and use that to predict cryptocurrency prices. This process requires finance experts, also requires much time from the experts and non-experts in this field to do the research needed for price prediction, which cannot be very accurate.

\subsection{PAPER STRUCTURE}

The remaining sections are arranged as follows. Section 2 examines similar research conducted in the blockchain area of study and gives a short summary of the swarm intelligence approaches and application. Section 4 gives details on the information collected and the experimental outcomes. Finally, the conclusion and future study are provided in Section 5.

\section{OVERVIEW OF RELATED WORK}

Metaheuristics begin with random training circuit solutions and, with time, improve the solution to minimise inaccuracies. In the SVM training phase, metaheuristic algorithms were applied, and it was stated that these methods outperformed a gradient algorithm when the issue was more challenging and multi-dimensional.

The collective actions of decentralised, self-organising, natural or artificial systems are swarm intelligence (SI). Swarm intelligence algorithms are the most popular method of resolving numerous NP-hard problems [7]. There are many Swarm intelligence algorithms like ant colony optimization (ACO) [8], particle swarm optimization (PSO) [9], bat algorithm [10], and Artificial Bee Colony (ABC) [11]. A variety of issues, including wireless sensor networks [12] [13] [14] [15], cloud computing [16] [17] [18], prediction, machine learning [17], MRI brain tumour classification [19], COVID-19 [20] [21] and global optimisation issues [22], have also been solved using SI approaches.

Analysis of earnings forecast of blockchain financial products based on PSO [23], in estimating the return rate of blockchain financial products, researchers attempted in this work to tackle a wide variety of problem areas, restrictions, and poor fitting effects of traditional methods. In this study, BP neural network with SVM and PSO was employed by researchers. The results demonstrate that their method is better than the other algorithms being evaluated in price prediction.

A study of financial investment based on the algorithm of the deep learning network was conducted [24]. The researchers tried to utilize the technology of deep learning to anticipate financial data for this study, with promising results. Finally, the ABC metaheuristic algorithm was used to optimise blockchain investment profile [25]. The researchers wanted to improve the security of asset securitisation and lower investment risk with their study. 
The results also show that the $\mathrm{ABC}$ algorithm has successfully optimised the investment portfolio and reduced the risk of decision-making.

\section{PROPOSED ALGORITHM}

Mirjalli presented the Sine Cosine Algorithm (SCA) technique in 2016, providing a mathematical model using the functions of the sine and cosine [26]. The SCA produces multiple initial, random solutions and enables them to swing towards or outwards the optimal solution. This approach additionally integrates several random and adaptable variables to emphasize the exploration and exploitation of the search space at different phases of optimization. And it was demonstrated that SCA is able effectively to explore different areas of a search area while avoiding local optima at a time when it converges to the global optimum and exploiting promising regions of an inquiry space during optimization.

In the exploration phase, an optimization algorithm rapidly combines the random solutions into a group of solutions with a large degree of diversity in order to find potentially interesting areas of the search space. However, throughout the exploitation process, there are increase changes in random solutions, and random differences are considerably less than within exploration.

The position update equations are as follows for exploration and exploitation:

$$
\begin{aligned}
& X_{i}^{t+1}=X_{i}^{t}+r_{1} \cdot \sin \left(r_{2}\right) \cdot\left|r_{3} P_{i}^{t}-X_{i}^{t}\right| \\
& X_{i}^{t+1}=X_{i}^{t}+r_{1} \cdot \operatorname{con}\left(r_{2}\right) \cdot\left|r_{3} P_{i}^{t}-X_{i}^{t}\right|
\end{aligned}
$$

Equation 1 position updating equations for the exploration and exploitation

Where $X_{i}^{t+1}$ symbolize the current position of the solution in the $i$-th dimension, at $t$-th iteration. Also $r_{r}$, $r_{2}$ and $r_{3}$ are three randomly generated numbers between 0 and $1, P_{i}^{t}$ symbolize the position of the $i$-th dimension, and $\|$ to symbolize that only the positive value will be used of $r_{3} P_{i}^{t}-X_{i}^{t}$.

These two equations are combined as follows:

$$
X_{i}^{t+1}=\left\{\begin{array}{l}
X_{i}^{t}+r_{1} \cdot \sin \left(r_{2}\right) \cdot\left|r_{3} P_{i}^{t}-X_{i}^{t}\right|, \quad r_{4}<0.5 \\
X_{i}^{t}+r_{1} \cdot \operatorname{con}\left(r_{2}\right) \cdot\left|r_{3} P_{i}^{t}-X_{i}^{t}\right|, r_{4} \geq 0.5
\end{array}\right.
$$

Equation 2 Combined equations of sine and cosine algorithm

Where $r_{4}$ shows a random number generated between 0 and 1.
As Equation 2 shows, SCA uses four primary parameters, and the parameters are accountable for the position of the destination. The search procedure, therefore, achieves a balance between solutions to coordinate well towards the optimal result. The cyclic architecture of the sine and cosine algorithm guarantees that the space between two solutions is exploited. In order to explore the search region, the solutions should be able to search throughout the area between their respective destinations. The sine and cosine algorithms are adjusted to do this. The sine and cosine range has to employ a solution to update its place outside the area between itself and another solution in order to guarantee the search area is investigated. Randomness can also be produced by finding a random number between 0 and $2 \pi$ for $r_{2}$ in Equation 2.

To balance the exploration phase with the exploitation phase, the SCA uses the following formula:

$$
r_{1}=a-t \frac{a}{T}
$$

Equation 3 balance the exploration and exploitation

Where $a$ symbolize a constant, $T$ symbolize the maximum number of iteration, and $t$ symbolize the current iteration number.

However, it was shown that metaheuristic exploration might be enhanced by doing original SCA tests using basic CEC unconstrained benchmarks. In order to solve this, the basic SCA was introduced to a simple exploration mechanism: the lowest solution from the population is replaced each time in the lower and upper boundaries of the search space by randomly generated individuals using the following equation:

$$
X_{m d}^{j}=L^{j}+\varnothing \cdot\left(U^{j}-L^{j}\right)
$$

Equation 4 update worst solution

Where $X_{r n d}^{j}$ is the $j$-th component of the random solution newly produced, $\phi$ in the other hand present a number chosen from a uniform distribution, and $U^{j}$ is the upper boundary, and $L^{j}$ is the lower boundary of the $j$-th parameter.

The proposed improved SCA metaheuristics are known as enhanced SCA (eSCA). Algorithm (1) displays pseudo-code. 


\section{Algorithm 1 - Pseudo-code of proposed eSCA}



\subsection{SUPPORT VECTOR MACHINE}

SVM is a technology for machine learning, developed by Vapnik and Cortes in 1995, founded on the VC dimension and the idea for structural risk reduction. It includes maximum interval hyperplanes, convex, slack variables and mercer's kernel. The technical theory is particularly ideal for small simple, high-dimensional and non-linear problems and has a significant capacity for generalization and machine learning [27].

SVM is used for two things: selecting the appropriate kernel function and fine-tuning their parameters. Searching for the best decision plane is an optimization challenge from a computational point of view. By using a non-linear transformation, the kernel function assists in the creation of linear decision planes. This section explains the foundations of SVM for classification problem.

Let us consider a dataset and its class labels, denoted by $S=x 1, \ldots x n$ and $G=y 1, \ldots y n$, respectively. SVM aims to find the best hyperplane $H$ to separate two data samples and create the most extensive interval $r$ between them. The ideal hyperplane $\mathrm{H}$ is represented as:

$$
W^{T} x+b=0
$$

Equation 5 Ideal hyperplane

Where $W$ indicates the weight vector and $b$ refers to the bias.

The problem has now been turned into an optimum problem of $W$ and $b$ as the following:

$$
\left\{\begin{array}{c}
\min _{\omega, b} r(\omega)=\frac{1}{2}\|\omega\| \\
y_{i}((\omega \cdot x)+b) \geq 1, i=1,2, \ldots, n
\end{array}\right.
$$

To meet the Karush Kuhn Tucker (KKT) criteria, the previous equation can be simplified by inserting Lagrange multipliers. The goal function is simplified to:

$$
\begin{gathered}
\min _{a} \frac{1}{2} \sum_{i=1}^{n} \sum_{j=1}^{n} y_{i} y_{j} a_{i} a_{j}\left(x_{i} \cdot x_{j}\right)-\sum_{j=1}^{n} a_{j} \\
\text { s.t. } \sum_{i=1}^{n} y_{i} a_{i}=0,0 \leq a_{i} \leq C
\end{gathered}
$$

According to the experimental results, the bigger the value of $C$, the bigger the separation interval, but the more significant the generalization risk. The ultimate classification function is as follows:

$$
f(x)=\operatorname{sgn}\left\{\left(\sum_{i=1}^{n} y_{i} a_{i} k\left(x_{i} \cdot x_{j}\right)\right)+b^{*}\right\}
$$

Equation 6 Ultimate classification function

By translating the nonlinear data into a high-dimensional feature space, kernel functions may be utilized to separate it linearly. The kernel function is defined as follows:

$$
K\left(x_{i}, x\right)=(\varphi(x i), \varphi(x))
$$

Equation 7 Kernel function

As a result, the final classification function can be expressed as:

$$
f(x)=\operatorname{sgn}\left\{\left(\sum_{t=1}^{n} y_{i} a_{i} k\left(x_{i} \cdot x_{j}\right)\right)+b\right\}
$$

Equation 8 Final classification function

The Gaussian kernel (radial basis function, RBF) is one of the most often used kernels for high-dimensional non-linear data, and it is expressed as the following:

$$
k(x, y)=\exp (-y\|x-y\|)^{2}
$$

Equation 9 Gaussian kernel

The classification performance of SVM is mostly determined by these two parameters, $\mathrm{C}$ and, which must be modified.

\section{EXPERIMENTAL SETUP AND ANALYSIS}

In the conducted experiments, the eSCA was tested with five years of daily prices from 2013 until 2018 for six types of cryptocurrency. Furthermore, this dataset includes open and close prices, also the highest and lowest price during the day (Table 1). 


\begin{tabular}{ll}
\hline Variable & Description \\
\hline Open Price & $\begin{array}{l}\text { The opening price of a certain } \\
\text { cryptocurrency on a given trading day. }\end{array}$ \\
\hline Close Price & $\begin{array}{l}\text { The closing price of a certain } \\
\text { cryptocurrency on a given trading day. }\end{array}$ \\
\hline Low Price & $\begin{array}{l}\text { The lowest price of a certain } \\
\text { cryptocurrency on a given trading day. }\end{array}$ \\
\hline High Price & $\begin{array}{l}\text { The highest price of a certain } \\
\text { cryptocurrency on a given trading day. }\end{array}$ \\
\hline
\end{tabular}

The training and testing dataset are provided in Table 2, with the number of training and testing simple for each cryptocurrency that has been used in this experiment. It should be highlighted that the number of training simples is different between the cryptocurrencies, but all of them have the same number of testing simples which is 364 .

Table 1 Description of variables

\begin{tabular}{ccccccc}
\hline & Training dataset & & & & Testing dataset \\
\hline $\begin{array}{c}\text { Crypto } \\
\text { currency }\end{array}$ & From & To & $\begin{array}{c}\text { Number of } \\
\text { samples }\end{array}$ & From & To & $\begin{array}{c}\text { Number of } \\
\text { simples }\end{array}$ \\
\hline BTC & 28/Mar/2013 & $16 / \mathrm{Jan} / 2017$ & 1388 & $17 / \mathrm{Jan} / 2017$ & $16 / \mathrm{Jan} / 2018$ & 364 \\
\hline ETH & $07 / \mathrm{Aug} / 2015$ & $16 / \mathrm{Jan} / 2017$ & 526 & $17 / \mathrm{Jan} / 2017$ & $16 / \mathrm{Jan} / 2018$ & 364 \\
\hline LTC & 28/Apr/2013 & $16 / \mathrm{Jan} / 2017$ & 1358 & $17 / \mathrm{Jan} / 2017$ & $16 / \mathrm{Jan} / 2018$ & 364 \\
\hline XRP & $04 / \mathrm{Aug} / 2013$ & $16 / \mathrm{Jan} / 2017$ & 1262 & $17 / \mathrm{Jan} / 2017$ & $16 / \mathrm{Jan} / 2018$ & 364 \\
\hline XEM & $01 / \mathrm{Aug} / 2015$ & $16 / \mathrm{Jan} / 2017$ & 657 & $17 / \mathrm{Jan} / 2017$ & $16 / \mathrm{Jan} / 2018$ & 364 \\
\hline XLM & $05 / \mathrm{Aug} / 2014$ & $16 / \mathrm{Jan} / 2017$ & 896 & $17 / \mathrm{Jan} / 2017$ & $16 / \mathrm{Jan} / 2018$ & 364 \\
\hline
\end{tabular}

Table 2 Dataset used for training and testing process

In Python environment, the SVM-eSCA model was constructed by leveraging popular keras machine library and scikit-learn tools. On the Nvidia 1080 with $8 \mathrm{~GB}$ of RAM 6X GPU tests are run. The experiment begins with observing and controlling performance measurements for different sorts of cryptocurrencies by classification.

Tables 3 and 4 illustrate the accuracy rate of the cryptocurrency market cap classifications. Table 3 illustrates several classifiers performance metrics, whereas Table 4 displays the support vector machine, support vector machine standard with optimized particle swarm optimization parameter selection and support vector machine standard with enhanced sine cosine algorithm.

\begin{tabular}{lcccccc}
\hline \multirow{2}{*}{ Classifiers } & \multicolumn{6}{c}{ Performance Accuracy (\%) } \\
\cline { 2 - 7 } & BTC & ETH & LTC & XRP & XEM & XLM \\
\hline ANN & 79.40 & 78.00 & 75.80 & 81.40 & 77.80 & 89.80 \\
\hline DL & 61.90 & 69.40 & 62.80 & 60.90 & 57.20 & 70.70 \\
\hline SVM & 78.90 & $\mathbf{9 5 . 5 0}$ & $\mathbf{8 2 . 4 0}$ & 70.00 & 47.70 & 58.70 \\
\hline \multirow{2}{*}{ BoostedNN } & 81.20 & 81.60 & 72.20 & $\mathbf{8 1 . 5 0}$ & 77.40 & $\mathbf{9 2 . 8 0}$ \\
\hline
\end{tabular}

Table 3 Classifiers performance accuracy

\begin{tabular}{lcccccc}
\hline \multirow{2}{*}{ Classifiers } & \multicolumn{6}{c}{ Performance Accuracy (\%) } \\
\cline { 2 - 7 } & BTC & ETH & LTC & XRP & XEM & XLM \\
\hline SVM [28] & 78.90 & 95.50 & 82.40 & 70.00 & 47.70 & 58.70 \\
\hline $\begin{array}{l}\text { Optimized } \\
\text { SVM-PSO [28] }\end{array}$ & 90.40 & $\mathbf{9 7 . 0 0}$ & $\mathbf{9 2 . 1 0}$ & 82.80 & 57.80 & 64.50 \\
\hline SVM-eSCA & 91.21 & $\mathbf{9 7 . 4 4}$ & $\mathbf{9 2 . 3 1}$ & $\mathbf{8 4 . 0 7}$ & $\mathbf{5 8 . 8 6}$ & $\mathbf{6 6 . 2 3}$ \\
\hline
\end{tabular}

Table 4 Comparison to find the best classifier

Several classifiers with the same data have been trained. The data sets were tested using the accuracy of classifications on this occasion. The identical set of data is used to compare all classifiers.

\section{CONCLUSION}

This paper proposes an enhanced SCA approach that was applied to optimize the SVM. The proposed method was tested on the cryptocurrency prediction problem, and compared to the standard SVM method and SVMPSO method. 
The conducted experiments clearly indicate that the proposed SCA approach obtained the best accuracy of all methods included in the analysis.

The future work in this domain will include testing of the other SI metaheuristics, in improved or hybridized versions on the same SVM optimization problem, and also the application of the eSCA method to other practical problems as well.

\section{REFERENCES}

[1] M. I. Aojia, S. Shaurya and Zhao, "Automated bitcoin trading via machine learning algorithms," URL: http://cs229. stanford. edu/proj2014/Isaacl\% 20Madan, vol. 20, 2015.

[2] Z. Aviv, "Bitcoin: under the hood," Communications of the ACM, vol. 58, no. 9, pp. 104-113, 2015.

[3] D. L. K. Chuen, L. Guo and Y. Wang, "Cryptocurrency: A new investment opportunity?," The Journal of Alternative Investments, vol. 20, no. 3, pp. 16-40, 2017.

[4] A. Heid, "Analysis of the cryptocurrency marketplace," Retrieved February, vol. 15, p. 104, 203.

[5] G. Bontempi, S. B. Taieb and Y.-A. L. Le Borgne, "Machine learning strategies for time series forecasting," European business intelligence summer school, pp. 62-77, 202.

[6] R. Farell, "An analysis of the cryptocurrency industry," 2015.

[7] G. Beni and J. Wang, "Swarm intelligence in cellular robotic systems," Robots and biological systems: towards a new bionics?, pp. 703-712, 1993.

[8] M. Dorigo, M. Birattari and T. Stutzle, "Ant colony optimization," IEEE computational intelligence magazine, vol. 1, no. 4, pp. 28-39, 2006.

[9] J. Kennedy and R. Eberhart, "Particle swarm optimization," Proceedings of ICNN'95-international conference on neural networks, vol. 4, pp. 1942-1948, 1995.

[10] X.-S. Yang, "A new metaheuristic bat-inspired algorithm," Nature inspired cooperative strategies for optimization (NICSO 2010), pp. 65-74, 2010.

[11] D. Karaboga and B. Basturk, "On the performance of artificial bee colony $(\mathrm{ABC})$ algorithm," Applied soft computing, vol. 8, no. 1, pp. 687-697, 2008.

[12] N. Bacanin, E. Tuba, M. Zivkovic, I. Strumberger and M. Tuba, "Whale Optimization Algorithm with Exploratory Move for Wireless Sensor Networks Localization," International Conference on Hybrid Intelligent Systems, pp. 328-338, 2019.

[13] M. Zivkovic, N. Bacanin, E. Tuba, I. Strumberger, T. Bezdan and M. Tuba, "Wireless Sensor Networks Life Time Optimization Based on the Improved
Firefly Algorithm," 2020 International Wireless Communications and Mobile Computing (IWCMC), pp. 1176-1181, 2020.

[14] M. Zivkovic, N. Bacanin, T. Zivkovic, I. Strumberger, E. Tuba and M. Tuba, "Enhanced Grey Wolf Algorithm for Energy Efficient Wireless Sensor Networks," 2020 Zooming Innovation in Consumer Technologies Conference (ZINC), pp. 87-92, 2020.

[15] M. Zivkovic, T. Zivkovic, K. Venkatachalam and N. Bacanin, "Enhanced Dragonfly Algorithm Adapted for Wireless Sensor Network Lifetime Optimization," Data Intelligence and Cognitive Informatics, pp. 803-817, 2021.

[16] N. Bacanin, T. Bezdan, E. Tuba, I. Strumberger, M. Tuba and M. Zivkovic, "Task scheduling in cloud computing environment by grey wolf optimizer," 2019 27th Telecommunications Forum (TELFOR), pp. 1-4, 2019.

[17] T. Bezdan, M. Zivkovic, M. Antonijevic, T. Zivkovic and N. Bacanin, "Environment, Enhanced Flower Pollination Algorithm for Task Scheduling in Cloud Computing," Machine Learning for Predictive Analysis, pp. 163-171, 2021.

[18] T. Bezdan, M. Zivkovic, E. Tuba, I. Strumberger, N. Bacanin and M. Tuba, "Multi-objective Task Scheduling in Cloud Computing Environment by Hybridized Bat Algorithm," International Conference on Intelligent and Fuzzy Systems, pp. 718-725, 2020.

[19] T. Bezdan, M. Zivkovic, E. Tuba, I. Strumberger, N. Bacanin and M. Tuba, "Glioma Brain Tumor Grade Classification from MRI Using Convolutional Neural Networks Designed by Modified FA," International Conference on Intelligent and Fuzzy Systems, pp. 955-963, 2020.

[20] M. Zivkovic, N. Bacanin, K. Venkatachalam, A. Nayyar, A. Djordjevic, I. Strumberger and F. AlTurjman, Sustainable Cities and Society, vol. 66, p. 102669, 2021.

[21] M. Zivkovic, K. Venkatachalam, N. Bacanin, A. Djordjevic, M. Antonijevic, I. Strumberger and T. A. Rashid, "Hybrid Genetic Algorithm and Machine Learning Method for COVID-19 Cases Prediction," Proceedings of International Conference on Sustainable Expert Systems: ICSES 2020, vol. 167, p. 169, 2021.

[22] I. Strumberger, E. Tuba, M. Zivkovic, N. Bacanin, M. Beko and M. Tuba, "Dynamic search tree growth algorithm for global optimization," Doctoral Conference on Computing, Electrical and Industrial Systems, pp. 143-153, 2019.

[23] W. Gao and C. Su, "Analysis of earnings forecast of blockchain financial products based on particle swarm optimization," Journal of Computational and Applied Mathematics, vol. 372, p. 112724, 2020. 
[24] M. Xie, H. Li and Y. Zhao, "Blockchain financial investment based on deep learning network algorithm," Journal of Computational and Applied Mathematics, vol. 372, p. 112723, 2020.

[25] Y. Deng, H. Xu and J. Wu, "Optimization of blockchain investment portfolio under artificial bee colony algorithm," Journal of Computational and Applied Mathematics, vol. 385, p. 113199, 2021.

[26] S. Mirjalili, "SCA: a sine cosine algorithm for solving optimization problems," Knowledge-based systems, vol. 96, pp. 120-133, 2016.

[27] C. Cortes and V. Vapnik, "Support-vector networks," Machine learning, vol. 20, no. 3, pp. 273297, 1995.

[28] N. A. Hitam, A. R. Ismail and F. Saeed, "An optimized support vector machine (SVM) based on particle swarm optimization (PSO) for cryptocurrency forecasting," Procedia Computer Science, vol. 163, pp. 427-433, 2019. 